\title{
Volcanic ash \& health: New chemical and isotopic approach
}

\author{
LUCIE SAUZÉAT, DAVID VOLLE, CLAUDE BEAUDOIN \\ AND MAUD BOYET
}

Université Clermont Auvergne

Presenting Author: lucie.sauzeat@uca.fr

Volcanic ash exposure may lead to significant risk. To date, damages on the respiratory and pulmonary system are the most evident toxic side effects although the causes of these symptoms remain unclear. Additionally, the effects on other organs remain largely under-explored, limiting our understanding of the longterm volcanic ash-related risk at the whole-body scale.

The metallome i.e., metal concentrations and isotopic compositions within the body, is suspected to be affected by volcanic ash exposure, having thus the potential for capturing some specificities of ash toxicity. The way and extent the metallome is affected and how these chemical deregulations correlate with pathophysiological dysfunctions is however poorly understood. Here, we adopt a multidisciplinary approach combining high precision chemical analyses (major and trace element concentrations) and $\mathrm{Cu}-\mathrm{Zn}$ isotope measurements coupled with histological and biochemical analyses in 9 organs and 2 biological fluids of mice (C57BL/6) exposed to volcanic ash.

We show that after 1 month of exposure, mice develop important metal homeostasis deregulations characterized by generalized heavy metal accumulation in the body associated to a significant and organ-specific $\mathrm{Cu}$ and $\mathrm{Zn}$ isotopic divergence observed in liver, kidney, urine and blood. These chemical variations are associated with early testicular defects and hepatic impairments that may, after prolonged exposure, seriously affect fertility and favor the emergence of liver diseases. Altogether, these results show that volcanic ash is an important metalenriched reservoir being highly toxic for the organism. $\mathrm{Cu}-\mathrm{Zn}$ isotope compositions are promising tools for monitoring the toxic effect of volcanic ash and identifying the main biological processes and vital functions vulnerable to long-term exposure. 